COVID-19 in the local area increased during this period. Transmission within halls of residence may have contributed to high positivity rates on campus. A high proportion of users reported symptoms of COVID-19 infection, despite this being an asymptomatic testing programme. Over a third of symptomatic cases were not isolating prior to receiving their result . These findings suggest that testing and isolating messaging may not be reaching the student population. Those with symptoms had a similar viral load to those without and may be similarly infectious.

\section{P55 LOW INCOME PARENTS' PERSPECTIVES AND EXPERIENCES OF ENGAGING WITH EARLY YEARS HEALTH PROFESSIONALS ABOUT FINANCIAL CHALLENGES AND INCOME MAXIMISATION}

${ }^{1}$ Flora Douglas*, ${ }^{1}$ Emma Maclver, ${ }^{2}$ Tracy Davis. ${ }^{1}$ School of Nursing, Midwifery and Paramedic Practice, Robert Gordon University, Aberdeen, UK; ${ }^{2}$ Public Health Directorate, NHS Grampian, Aberdeen, UK

\subsection{6/jech-2021-SSMabstracts. 143}

Background Since the introduction of the Child Poverty Act (2017) in Scotland, all health visitors, midwives and family nurses in Scotland are expected to screen and offer a financial advice referral to at-risk pregnant women and parents/carers of families with children under five in Scotland. The so-called 'Financial Inclusion Pathway' (FIP) emerged in 2019 as one of a number of strategies intended to tackle child poverty. At this early implementation stage, little is known about parents' perspectives about the acceptability or impact of this initiative in relation to its aim.

Methods In 2020, low income parents with young children living in Aberdeen City were invited to take part in a qualitative study that aimed to determine any challenges they might face engaging with the FIP policy in practice, and their perspectives about financial discussions with professionals that could help to increase household incomes through this approach.One-to-one semi-structured telephone interviews lasting between 30-40 minutes took place during July and August 2020. Interviews were fully transcribed and thematically analysed.

Results Ten women, ranging from ages 20-41 years. Each participant had between one and five child(ren), ranging in age from 2 to 18 years and all had one child under school-age. All lived in multiply deprived postcode areas within Aberdeen City. Three main themes emerged: i. difficulties associated with discussing financial concerns; ii. how to talk about the issue; iii. intervention utility. Within each of these three main themes, nine-sub themes were apparent, ranging from those indicating potential unintended negative consequences, as well as intervention benefits.

Discussion Most participants considered health visitors to be a potentially good source of help about financial challenges; they were less clear about midwives' role here. However, parents' perceptions of the problems they may face associated with disclosing financial difficulties to health professionals, is a distinct barrier to conversations that could lead to a financial advice referral. Given the levels of unclaimed benefit in the UK, this is important to note. Establishing trust and rapport, careful and sensitive enquiry, and positive framing of financial maximisation were recommended to aid discussion of financial challenges. These findings are important given the current and predicted economic impact of the COVID-19. The study is limited by its sample size and recruitment site, but provides valuable insights to inform a larger scale investigation. The study also indicates the importance of understanding health professionals' associated views and experiences.

\section{P56 CHILDREN'S HEALTH IN CARE IN SCOTLAND (CHICS) STUDY - POPULATION-WIDE LINKED ADMINISTRATIVE DATA ANALYSIS COMPARING HEALTH OUTCOMES FOR CARE EXPERIENCED AND CHILDREN IN THE GENERAL POPULATION, 2009-2016}

${ }^{1}$ Mirjam Allik*, 'Denise Brown, ${ }^{1}$ Alastair Leyland, ${ }^{2}$ Marion Henderson. ${ }^{1}$ SPHSU, University of Glasgow, Glasgow, UK; ${ }^{2}$ Social Work and Social Policy, University of Strathclyde, Glasgow, UK

\subsection{6/jech-2021-SSMabstracts. 144}

Background There is little quantitative evidence on how the health of care experienced children compares to children in the general population in Scotland or the UK. Invariably, the evidence that is available suggests that care experience is related to poorer health, but often this is based on small sample sizes or without comparison to children who have not been in care. The CHiCS study was set up to provide the first population-wide evidence on health outcomes (mortality, hospitalisation, prescribing and pregnancy rates) of care experienced children (CEC) compared to children in the general population (CGP).

Methods We undertook an individual level linkage of the Children Looked After in Scotland (CLAS) return to the Pupil Census (PC), Scottish Morbidity Records (SMR00, 01, 02, 04), Accidents and Emergency data, Prescribing Information System, and Death and Birth Registrations from August 2009 to July 2016. The CEC cohort was defined as all children who were on the 2009/2010 CLAS return and the CGP cohort was defined as children who were on the 2009 PC but had not been in care. Age-standardized rates by area deprivation will be calculated for various outcomes for both cohorts to provide the first national evidence on the health of looked after children. Event history analysis will be used on matched cohorts to investigate the impact of placement histories and parental socioeconomic status (SES) on health.

Results The data became available for analysis in December 2020. The initial results show that CEC have, on average, lower SES and live in areas of higher deprivation, they also experienced higher average rates of mortality, prescriptions, and all types of hospitalisations during the study period (not adjusted). There are also substantial differences in the types of prescriptions and reasons for hospital admission between the cohorts, e.g. CEC are more likely to be prescribed antidepressant and ADHD medication and have almost three times as many psychiatric outpatient appointments (not adjusted). Additional results will be submitted before the June 28th deadline. We expect the results adjusted for confounders to show substantial differences in the frequency and reasons for contact with health services between the two cohorts.

Results Adverse childhood experiences have a profound negative impact on health and these effects last long into adulthood. Identifying how care experiences are related to health will provide evidence to develop interventions for health improvement and to reduce inequalities. Interventions in childhood are among the most effective in improving long-term health and are cost-effective. 